\title{
锂离子电池硅/碳纳米管/石墨烯自支撑负极材料研究
}

\author{
白雪君 ${ }^{1}$, 刘 婵 ${ }^{1}$, 侯 敏 ${ }^{1}$, 王 彪 ${ }^{2}$, 曹 辉 ${ }^{1,3}$, 付俊杰 ${ }^{4}$
}

(1. 上海航天电源技术有限责任公司，上海 201615; 2. 东华大学 材料科学与工程学院，上海 201620; 3. 上海空间电源研 究所, 上海 201216; 4. 中石化海洋石油工程有限公司上海物探分公司, 上海 201208)

摘 要: 通过真空驱动自组装法及蒸汽处理得到结构疏松的硅/碳纳米管/石墨烯自支撑负极材料(Si/CNTs/GP)。纳米 硅颗粒 $(50 \mathrm{~nm})$ 为活性物质，均匀分布在石墨烯片层结构中间; 石墨烯作为碳基体，通过自组装构筑形成二维导电 网络; 碳纳米管(CNTs)具有超高导电性和良好的力学强度，它通过吸附作用均匀分布在石墨烯基体上形成导电的 支撑网络结构。经过蒸汽处理后，石墨烯层间距明显增大，层与层之间不再是紧密堆叠的结构，而是形成一种疏 松、褶皱、内部空隙丰富的片层结构。 $\mathrm{Si} / \mathrm{CNTs} / \mathrm{GP}$ 负极材料中丰富的内部空穴和贯穿孔洞，提供了材料很高的比 表面积, 能有效提高电解液对材料的浸润性，极大缩短了离子传输距离。同时这些内部空穴也有效缓冲硅充放电时 的体积膨胀, 提高了材料的结构稳定性和电化学性能。该负极材料在 $4 \mathrm{~A} / \mathrm{g}$ 的大电流密度下容量维持在 $600 \mathrm{mAh} / \mathrm{g}$, 表现出良好的大电流循环稳定性能。

关 键 词: 石墨烯; 硅; 锂离子电池; 自支撑; 蒸汽处理

中图分类号: TM911; O613 文献标识码: A

\section{Silicon/CNTs/Graphene Free-standing Anode Material for Lithium-ion Battery}

\author{
BAI Xue-Jun ${ }^{1}$, LIU Chan ${ }^{1}$, HOU Min ${ }^{1}$, WANG Biao ${ }^{2}$, CAO Hui ${ }^{1,3}$, FU Jun-Jie ${ }^{4}$
}

(1. Shanghai Aerospace Power Technology Co., LTD, Shanghai 201615, China; 2. College of Material Science and Engineering, Donghua University, Shanghai 201620, China; 3. Shanghai Institute of Space Power Source, Shanghai 201216, China; 4. Shanghai Geophysical Branch, Sinopec Offshore Oilfield Services Company, Shanghai 201208, China)

\begin{abstract}
Porous 2D Silicon/CNTs/Graphene free-standing composites were prepared via a solution-based self-assembly process with vapor post-treatment and applied as anodes for lithium-ion batteries. Silicon nanoparticles $(\sim 50 \mathrm{~nm})$ as active materials were uniformly embedded between graphene sheets without agglomeration. Graphene was used as electrical conductive carbon matrix to form a 2D conductive network for electrons. CNTs, with high electrical conductivity and mechanical strength, formed into a scaffold along with graphene to enhance the conductivity and mechanical properties of the carbon matrix. After vapor post-treatment, CNTs supported graphene film transferred from a tightly stacked film to a loose packed porous film. The porous structure of this free-standing composite anode provides large internal space to accommodate volumetric changes and provide abundant channels for diffusion of $\mathrm{Li}^{+}$, fast electron transport and easy penetration of electrolyte. The composites exhibite an outstanding rate performance and cyclic stability, delivering a capacity of $600 \mathrm{mAh} / \mathrm{g}$ at 4/A g and $1010 \mathrm{mAh} / \mathrm{g}$ after $100 \mathrm{cycles}$ at $0.1 \mathrm{~A} / \mathrm{g}$. They also show high structure and mechanical stability after cycle test. It provides an exciting pathway to the rational design and fabrication of silicon anode and 2D graphene matrix for applications in lithium-ion batteries.
\end{abstract}

Key words: graphene; silicon; lithium-ion battery; free-standing; vapor post-treatment 
锂离子电池是重要的储能设备, 具有高能量密 度、大输出功率、高开路电压、低自放电率等优异 特性, 在电动汽车、便携式电子产品等领域占领了 大量市场。尤其是随着电动汽车的快速发展, 对锂 离子电池容量、能量密度和快速充放电能力提出了 越来越高的要求 ${ }^{[1-3]}$ 。目前商业化锂离子电池负极材 料为石墨, 其容量密度仅为 $372 \mathrm{mAh} / \mathrm{g}$, 在很大程 度上限制了锂离子电池向高容量方向的发展。因此, 开发性能稳定的高容量负极材料成为锂离子电池研 究的一个重要方向 ${ }^{[4]}$ 。硅作为地球上第二丰富的元 素, 具有高达 $4200 \mathrm{mAh} / \mathrm{g}$ 的理论储锂容量, 且硅的 电压平台较高, 不会在负极表面形成锂枝晶, 具有 很高的安全性能 ${ }^{[5]}$, 因此硅成为目前研究最热的锂 离子电池负极材料之一。目前硅负极材料主要以硅 单质、硅的氧化物、硅复合物等形式存在 ${ }^{[6-9]}$, 但是 硅负极在嵌/脱锂的过程中会发生严重的体积膨胀和 收缩, 体积变化率高达 $300 \%$, 这会使材料内部受到 巨大的应力作用而导致结构发生严重的破坏甚至粉 化, 使材料失去电接触, 导致容量的迅速衰减 ${ }^{[10-11]}$ 。

为了克服这一问题, 近年来研究人员进行了大 量的研究工作。通过制备硅纳米颗粒 ${ }^{[12-14]}$ 、硅纳米 线 ${ }^{[15-18]}$ 、硅纳米管 ${ }^{[19-21]}$ 、多孔硅 ${ }^{[22-24]}$ 等来缩小硅材 料的尺寸, 降低体积膨胀时产生的应力, 提高硅的 结构稳定性, 同时还可以缩短锂离子的传输距离, 提升硅负极的电化学性能。在此基础上引入导电缓 冲基体, 在提高硅材料导电性的同时又能够利用基 体的弹性、稳定性等性能来有效控制和缓解硅材料 的体积变化, 维持材料整体结构的完整性, 如无定 形碳包覆、石墨烯支撑、三维碳网络等 ${ }^{[17,25-30]}$ 。结 合上述两种改性方法, 本工作以纳米硅颗粒为活性 物质, 石墨烯和碳纳米管(CNTs) 为导电缓冲基体, 制备复合硅负极。并通过 XRD、SEM 和充放电测 试等研究该材料的结构、形貌和电化学性能, 以期 得到比容量高、倍率性能好、循环稳定的锂离子电 池负极材料。

\section{1 实验方法}

\section{1 石墨烯/硅自支撑材料的制备}

采用改性 Hummers 法 ${ }^{[28]}$ 制备氧化石墨烯(GO), 得到浓度为 $0.5 \mathrm{wt} \%$ 的 $\mathrm{GO}$ 水溶液。

将 $5 \mathrm{~mL} \mathrm{GO}$ 水溶液与 $20 \mathrm{~mL}$ 去离子水混合并超 声分散 $10 \mathrm{~min}$, 然后加入 $126 \mathrm{mg} \mathrm{NaCl}$ 和 $1.3 \mathrm{~mL}$ 聚 二烯丙基二甲基氯化铵 (PDDA, $\mathrm{Mw}<100000$, $35 \mathrm{wt} \%$ ) 水溶液, 强烈搅拌 $10 \mathrm{~min}$ 使反应物混合均
匀。将整个反应溶液加热至 $90^{\circ} \mathrm{C}$ 并反应 $5 \mathrm{~h}$, 同时 强烈搅拌。去离子水洗涤去除杂质后, 得到 $0.5 \mathrm{wt} \%$ 的 PAAD-GO 水溶液。将 $10 \mathrm{mg} \mathrm{Si}(30 \sim 50 \mathrm{~nm}, \mathrm{US}$ Research Nanomaterials)、2.5 mg 或 $5 \mathrm{mg} \mathrm{CNTs}(-\mathrm{OH}$ 基团改性, US Research Nanomaterials)与 $3 \mathrm{~mL}$ PAAD-GO 水溶液超声混合 $30 \mathrm{~min}$ 后, 将混合液置 于 $\mathrm{AAO}$ 滤膜, 在真空驱动下自组装形成 $\mathrm{Si} / \mathrm{CNTs} / \mathrm{GO}$ 膜。在密闭的 $20 \mathrm{~mL}$ 玻璃瓶中加入 $5 \mathrm{~mL}$ 乙醇, 将 $\mathrm{Si} / \mathrm{CNTs} / \mathrm{GO}$ 膜置于不锈钢网上, $80^{\circ} \mathrm{C}$ 加热 $1 \mathrm{~h}$, 利用乙醇蒸汽促使石墨烯层间距扩张, 得到结构 疏松的材料。然后再将膜置于石英管中进行热处理, 在氩气气氛下进行还原, 气体流速为 $10 \mathrm{~cm}^{3} / \mathrm{min}$ 。温 度以 $2^{\circ} \mathrm{C} / \mathrm{min}$ 的速率由室温升至 $92^{\circ} \mathrm{C}$, 保温 $10 \mathrm{~h}$, 随 后再以 $5^{\circ} \mathrm{C} / \mathrm{min}$ 的速率升至 $800^{\circ} \mathrm{C}$ 并保温 $2 \mathrm{~h}$, 最后 自然冷却至室温, 得到膜状负极材料 $\mathrm{Si} / \mathrm{CNTs} / \mathrm{GP}-1$ (或-2), 制备过程中材料结构变化示意图见图 1。

作为对比样品, 直接将 $10 \mathrm{mg} \mathrm{Si}$ 与 $3 \mathrm{~mL}$ PAAD-GO 水溶液超声混合 $30 \mathrm{~min}$ 后真空抽滤形成 $\mathrm{Si} / \mathrm{GO}$ 膜, 再将其置于石英管中进行热还原得到不 含 $\mathrm{CNTs}$ 的 $\mathrm{Si} / \mathrm{GP}$ 负极材料。然后直接将 $3 \mathrm{~mL}$ PAAD-GO 水溶液抽滤成膜, 再置于石英管中进行 热还原得到 GP 膜状材料。

\section{2 性能测试}

采用激光粒度仪(Malvern, Zetasizer Nano)对样 品表面带电性进行分析。采用 X 射线衍射仪(XRD, Rigaku D/max $2550 \mathrm{~V}, \mathrm{Cu}-\mathrm{K} \alpha, \lambda=0.154056 \mathrm{~nm}$ ) 对材 料物相进行分析。采用拉曼光谱仪(Raman, Horiba $\mathrm{T} 64000,514.5 \mathrm{~nm}$ 波长)和 $\mathrm{X}$ 射线光电子能谱(XPS,

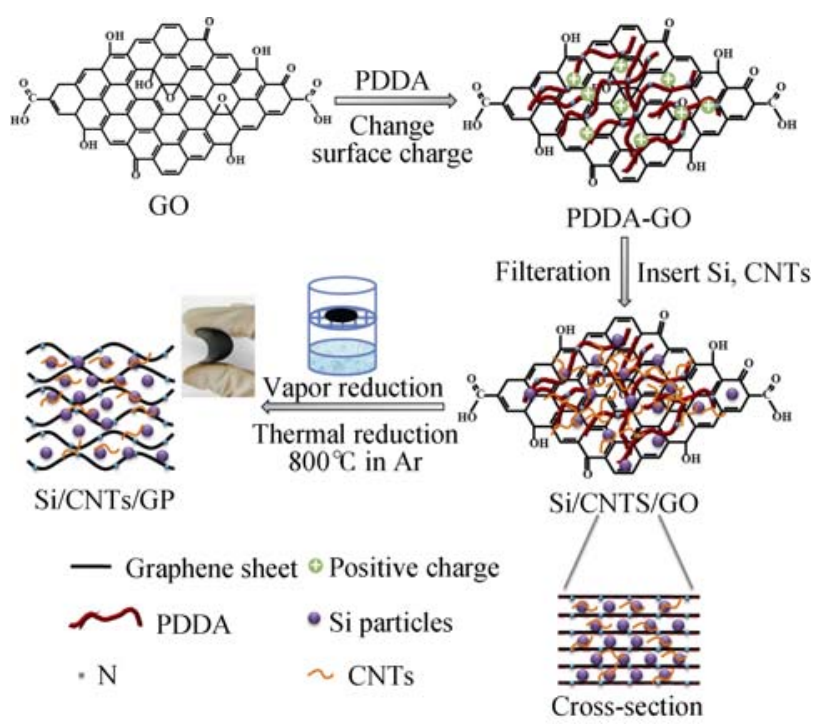

图 $1 \mathrm{Si} / \mathrm{CNTs} / \mathrm{GP}$ 负极材料制备过程中结构变化流程图

Fig. 1 Schematic illustration of synthesis of $\mathrm{Si} / \mathrm{CNTs} / \mathrm{GP}$ composites 
Thermo Scientific ESCALAB 250Xi, Al Ka, 1486.6 e) 对材料的组分进行分析。利用热重分析仪(TGA, Netzsh TG 209 F1)对样品的组分含量进行分析, 空气气氛 $(20 \mathrm{~mL} / \mathrm{min})$, 升温速率为 $15^{\circ} \mathrm{C} / \mathrm{min}$, 温 度范围 $25 \sim 800^{\circ} \mathrm{C}$ 。采用场发射扫描电镜(FESEM, Hitachi SU8030)对材料表面形貌进行观察，并利 用附件能量色散谱(EDS)对样品表面成分进行分 析。电池循环测试之后的电极在氩气气氛手套箱 中用碳酸二甲酯冲洗干净并自然干燥后再进行 FESEM 测试。

\section{3 电化学性能测试}

负极材料的电化学性能通过组装成 CR2032 型 扣式半电池进行测试。将制备得到的负极材料直接 裁剪成直径为 $1 \mathrm{~cm}$ 的圆片作为工作电极, 锂片作为 对电极, Celgard2400 为隔膜, $\mathrm{EC} / \mathrm{DMC}(1: 1)-\mathrm{LiPF}_{6}$ (1 mol/L) 为电解液 (BASF Corporation) 进行组装。整 个组装过程在氩气气氛手套箱中进行, 其中水、氧 含量均在 $10 \times 10^{-6}$ 以下。

采用电池测试系统(Arbin BT2000)对电池的循 环充放电容量、稳定性等进行测试。测试电压为 2.0 0.01 V(vs Li/ $\left./ \mathrm{Li}^{+}\right)$, 测试时活性物质质量分别为 $\mathrm{Si} / \mathrm{GP}$ 和 $\mathrm{Si} / \mathrm{CNTs} / \mathrm{GP}$ 材料的质量。采用电化学工作 站(Autolab PGSTAT128N) 对电池循环伏安曲线(CV) 和交流阻抗 (EIS) 进行测试。CV 测试电压为 $2.0 \sim 0.01 \mathrm{~V}\left(v s \mathrm{Li} / \mathrm{Li}^{+}\right)$, 电压扫速为 $0.1 \mathrm{mV} / \mathrm{s}$ 。 EIS 测 试电压为 $0.6 \mathrm{~V}$, 电压幅度为 $10 \mathrm{mV}$, 频率为 $10^{6} \sim$ $0.01 \mathrm{~Hz}$ 。

\section{2 结果与讨论}

\section{1 氧化石墨烯表面电性调节}

静电吸附作用对纳米材料微观结构的形态有着 关键影响, 尤其影响 $\mathrm{Si}$ 和 CNTs 在石墨烯中的分散 均匀性。本研究采用的 $\mathrm{Si}$ 和 CNTs 表面带有羟基基 团, 两者在水溶液中呈负电性。因此当 GO 在水溶 液中带正电时, 三者间才会存在较强的静电吸附作 用。图 2 为 $\mathrm{GO}$ 和 PDDA-GO 水溶液在不同 $\mathrm{pH}$ 下 的 Zeta 电位。当 $\mathrm{pH}$ 在 0 9 之间时, $\mathrm{GO}$ 水溶液的电 位始终在 0 以下, 带负电荷。这是由 GO 表面的羟 基和羧基等官能团的电离引起的。而经过 PDDA 改 性后的 $\mathrm{GO}$ 水溶液呈正电性。PDDA 中 $\mathrm{N}^{+}$基团与 $\mathrm{GO}$ 中的环氧基团反应, 促使其碳环的开环反应, 使 PDDA 中 $\mathrm{N}^{+}$基团嵌入 $\mathrm{GO}$ 的碳平面中, 因此 $\mathrm{GO}$ 在水溶液中呈正电性 ${ }^{[31]}$ 。同时 PDDA 主链上的不饱 和 $\mathrm{C}=\mathrm{C}$ 键与 $\mathrm{GO}$ 碳环平面之间的 $\pi-\pi$ 作用也会促使

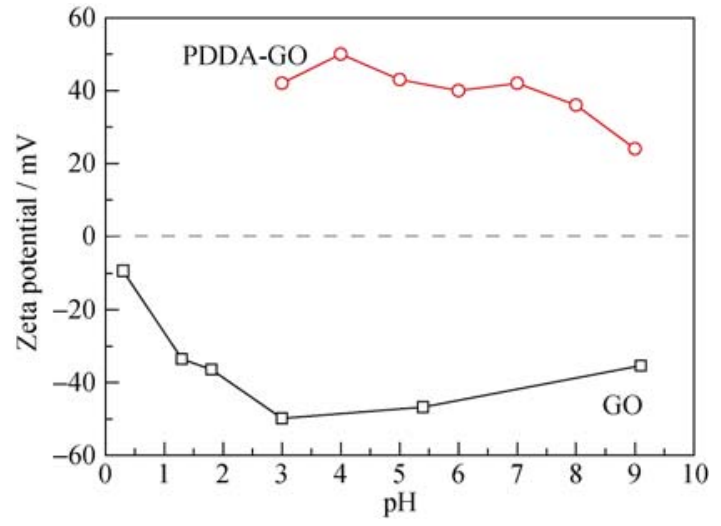

图 2 氧化石墨烯复合 PDDA 前、后在不同酸碱度下的 Zeta 电位

Fig. 2 Zeta potential of GO and PDDA-GO at different $\mathrm{pH}$

$\mathrm{GO}$ 由负电性转为正电性 ${ }^{[32]}$ 。因此在中性环境下, PDDA-GO 与 $\mathrm{Si} 、 \mathrm{CNTs}$ 具有相反电性, 会存在较强 的静电吸附作用, 有利于 $\mathrm{Si} 、 \mathrm{CNTs}$ 在 PDDA-GO 上 均匀附着, 得到分散良好的负极材料。

\section{2 石墨烯/硅自支撑材料的物相及形貌分析}

图 3 为不同样品的 XRD 图谱, 从图中可以看出: 研究采用的 $\mathrm{Si}$ 为晶体结构, 具有明显的 XRD 特征 峰。CNTs 在 $2 \theta=26^{\circ}$ 和 $45^{\circ}$ 左右有两个明显的峰, 对 应于石墨结构的(002)和(101)晶面。经过复合之后, $\mathrm{Si} / \mathrm{CNTs} / \mathrm{GO}$ 同时具有 $\mathrm{Si} 、 \mathrm{CNTs} 、 \mathrm{GO}$ 的特征峰，证 明三者被成功复合，且在复合过程中物相没有发生 明显变化。经过热还原后的 $\mathrm{Si} / \mathrm{CNTs} / \mathrm{GP}, \mathrm{GO}$ 的特征 峰完全消失，(002)晶面的特征峰更尖锐更明显，且 在 $2 \theta=20^{\circ} \sim 30^{\circ}$ 之间出现一个明显宽峰, 说明 GO 被 还原并具有一定的石墨化结构，但无定形碳的结构 依旧存在。Si/GP 样品中没有 $\mathrm{CNTs}$, 因此 $2 \theta=26^{\circ}$ 处 的衍射峰为宽峰。

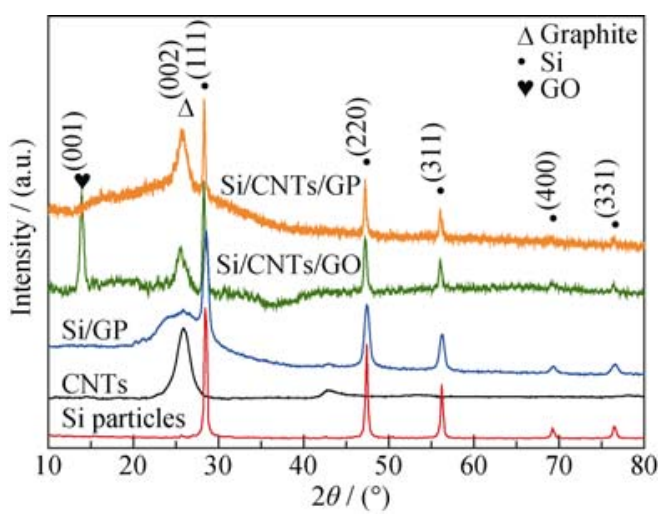

图 $3 \mathrm{Si} 、 \mathrm{CNTs} 、 \mathrm{Si} / \mathrm{GP} 、 \mathrm{Si} / \mathrm{CNTs} / \mathrm{GO}$ 和 $\mathrm{Si} / \mathrm{CNTs} / \mathrm{GP}$ 负极材 料的 XRD 图谱

Fig. 3 XRD patterns of $\mathrm{Si}, \mathrm{CNTs}, \mathrm{Si} / \mathrm{GP}, \mathrm{Si} / \mathrm{CNTs} / \mathrm{GO}$, and $\mathrm{Si} / \mathrm{CNTs} / \mathrm{GP}$ composite film 
为了进一步分析样品中碳材料的结构, 对各 个阶段样品进行拉曼光谱测试。如图 4 中所示, CNTs 在 1300、1594、2700 $\mathrm{cm}^{-1}$ 三处有明显特征 峰, 分别为 $D 、 G 、 2 D$ 峰。 $D$ 峰对应碳材料中的 无序结构, G 峰和 2D 峰对应碳材料中的有序石墨 化结构。因此 CNTs 中的 G 峰强度高于 D 峰, 且 具有明显的 $2 \mathrm{D}$ 峰。 $\mathrm{Si} / \mathrm{GP}$ 样品中也能看到 $2 \mathrm{D}$ 峰, 但形状为宽峰, 且 $D 、 G$ 峰强度基本一致, 说明该 还原方法能够有效还原 GO, 且得到具有一定石 墨化结构的石墨烯。与 $\mathrm{Si} / \mathrm{GP}$ 相比, $\mathrm{Si} / \mathrm{CNTs} / \mathrm{GP}$ 中 $\mathrm{G}$ 峰的强度更高, 2D 峰明显, CNTs 的存在对负极 材料的碳结构产生了明显影响。而 $200 \sim 500 \mathrm{~cm}^{-1}$ 之间的两个小峰对应于 $\mathrm{Si}$ 的 LA 和 TO 峰, $\mathrm{Si}$ 在整 个制备过程中结构稳定。

通过 XPS 测试可以进一步研究样品中所含元素 及其存在形式。由于 PDDA 的引入，图 5(a)中 $\mathrm{Si} / \mathrm{CNTs} / \mathrm{GO}$ 样品中除了 $\mathrm{Si} 、 \mathrm{C} 、 \mathrm{O}$ 元素外还含有少 量的 $\mathrm{Cl}$ 。热还原后, $\mathrm{Si} / \mathrm{CNTs} / \mathrm{GP}$ 中的 $\mathrm{Cl}$ 元素被去除, 且 $\mathrm{C}$ 峰强度有所提高。图 5(b)中不同阶段的样品的 $\mathrm{C}$ 峰经过拟合处理后可以被分为三个峰: 284.7、

$286.3 、 288.2 \mathrm{eV}$, 分别对应 $\mathrm{C}-\mathrm{C} 、 \mathrm{O}-\mathrm{C}-\mathrm{O} / \mathrm{C}-\mathrm{O} / \mathrm{C}-\mathrm{N}$ 、 $\mathrm{C}=\mathrm{O}$ 基团。CNTs 中 $\mathrm{C}$ 元素绝大部分以 $\mathrm{C}-\mathrm{C}$ 键形式 存在, 表面只有少量的羟基基团。因此, $\mathrm{Si} / \mathrm{CNTs} / \mathrm{GO}$ 的 $\mathrm{C}$ 谱与 $\mathrm{GO}$ 相比, $\mathrm{C}-\mathrm{C}$ 峰的强度更高一些。而经 过热还原后的 $\mathrm{Si} / \mathrm{CNTs} / \mathrm{GP}$ 和 $\mathrm{Si} / \mathrm{GP}$ 中 $\mathrm{O}-\mathrm{C}-\mathrm{O} / \mathrm{C}-\mathrm{O} /$ $\mathrm{C}-\mathrm{N} 、 \mathrm{C}=\mathrm{O}$ 峰强度明显下降, 说明热处理法可以在 很大程度上还原 GO。PDDA 的使用在反应体系中 引入了 $\mathrm{N}$ 元素, 如图 5(c)。 $\mathrm{Si} / \mathrm{CNTs} / \mathrm{GO}$ 中的 $\mathrm{GO}$ 经 过 PDDA 改性后, 在 399.5 和 $402.1 \mathrm{eV}$ 处两有明显 的 $\mathrm{N}$ 峰, 分别对应少量的吡咯型 $\mathrm{N}$ 和大量的含 $\mathrm{N}$ 官
能团。 $\mathrm{Si} / \mathrm{CNTs} / \mathrm{GO}$ 中的 $\mathrm{N}$ 元素大部分以含 $\mathrm{N}$ 官能 团的形式存在于碳环平面之外 ${ }^{[33]}$, 这也与 PDDA-GO 的带电性相符。经过热还原之后, $\mathrm{Si} / \mathrm{CNTs} / \mathrm{GP}$ 中的 $\mathrm{N}$ 以四种形式存在, 另外两个峰对 应于 $398.3 \mathrm{eV}$ 处的吡啶型 $\mathrm{N}$ 和 $401 \mathrm{eV}$ 处的四价 $\mathrm{N}$ 。 热还原过程中，碳环表面存在的含 $\mathrm{N}$ 官能团与 $\mathrm{GO}$ 上的含氧基团反应，取代其五圆环、六圆环中的 $\mathrm{C}$, 成功嵌入石墨烯的碳环结构中。这种 $\mathrm{N}$ 掺杂结构有 利于提高石墨烯的导电性 ${ }^{[34-35]}$ 。由于 $\mathrm{Si}$ 在空气及受 热条件下不稳定，实验利用 XPS 对 $\mathrm{Si}$ 热还原前后的 存在形式进行研究, 如图 5(d)所示。 $\mathrm{Si} / \mathrm{CNTs} / \mathrm{GO}$ 和 $\mathrm{Si} / \mathrm{CNTs} / \mathrm{GP}$ 中的 $\mathrm{Si}$ 都有三种形式, $\mathrm{Si}^{0}(99.8 \mathrm{eV}) 、 \mathrm{Si}^{2+}$ $(101.8 \mathrm{eV}) 、 \mathrm{Si}^{4+}(103.6 \mathrm{eV})$ 。经过热还原后的 $\mathrm{Si} / \mathrm{CNTs} / \mathrm{GP}$ 中的 $\mathrm{Si}^{2+}$ 和 $\mathrm{Si}^{4+}$ 含量略有增加, 但主要 存在形式还是 $\mathrm{Si}^{0}$ 。

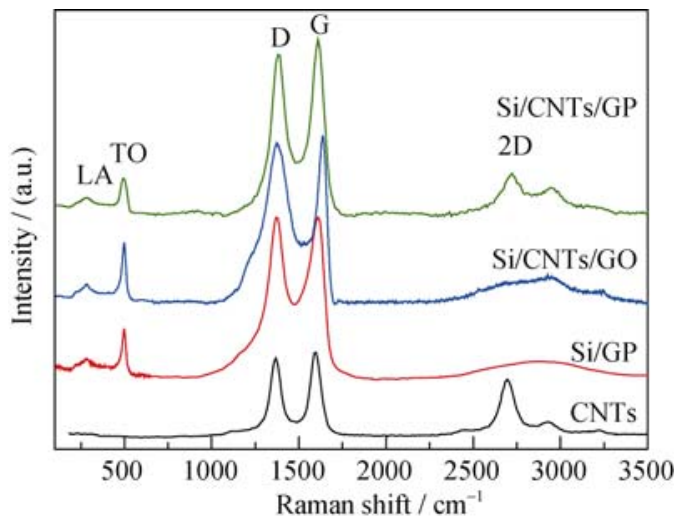

图 $4 \mathrm{CNTs} 、 \mathrm{Si} / \mathrm{GP} 、 \mathrm{Si} / \mathrm{CNTs} / \mathrm{GO}$ 和 $\mathrm{Si} / \mathrm{CNTs} / \mathrm{GP}$ 负极材料的 拉曼图谱

Fig. 4 Raman spectra of CNTs, Si/GP, Si/CNTs/GO and $\mathrm{Si} / \mathrm{CNTs} / \mathrm{GP}$ composite film
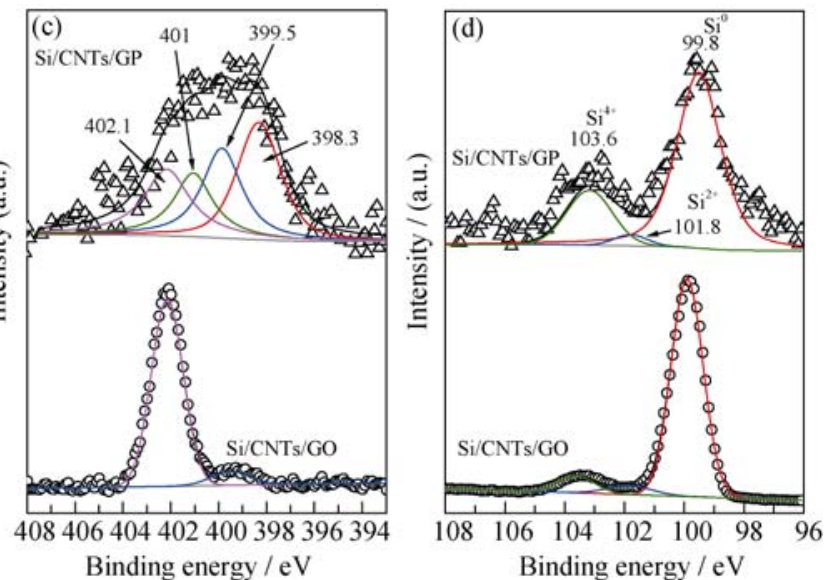

图 $5 \mathrm{CNTs} 、 \mathrm{Si} / \mathrm{GP} 、 \mathrm{Si} / \mathrm{CNTs} / \mathrm{GO}$ 和 $\mathrm{Si} / \mathrm{CNTs} / \mathrm{GP}$ 负极材料的 XPS 图谱

Fig. 5 XPS spectra of CNTs, Si/GP, Si/CNTs/GO, and Si/CNTs/GP composite (a) Survey spectra; (b) High resolution C1s spectra; (c) High resolution N1s spectra; (d) High resolution Si2p spectra 
作为锂离子电池负极材料, 各组分的含量对其 电化学性能都有很大影响, 通过热重分析法测量负 极材料中 $\mathrm{Si}$ 的含量。如图 6 所示, 在空气气氛下升 温至 $800^{\circ} \mathrm{C}, \mathrm{Si}$ 的质量略有增长, 为 $7 \mathrm{wt} \%$, 是由 $\mathrm{Si}$ 部分氧化引起的。而 GP 高温下与氧气反应并以气 体形式排出, 其剩余质量接近 $2 \mathrm{wt} \%$ 。在此基础上, 根据三种不同负极材料的剩余质量计算可得 $\mathrm{Si}$ 含 量。 $\mathrm{Si} / \mathrm{GP}$ 和 $\mathrm{Si} / \mathrm{CNTs} / \mathrm{GP}-(1,2)$ 负极材料的 $\mathrm{Si}$ 含量 分别为 $47 \mathrm{wt} \% 、 41 \mathrm{wt} \% 、 38 \mathrm{wt} \%$ 。

从图 7 可以看出, $\mathrm{Si}$ 颗粒尺寸为 $50 \mathrm{~nm}$ 左右, 且 颗粒尺寸均匀, 呈单分散无二次团聚, CNTs 长度约 为 $0.5 \sim 2 \mu \mathrm{m}$ 。 $\mathrm{Si}$ 和 CNTs 在材料中分布均匀, 无明 显团聚的现象, 这是由于 GO 经过 PDDA 改性后呈 正电性, 与 $\mathrm{Si}$ 和 $\mathrm{CNTs}$ 之间存在良好的静电吸附作 用。从三种样品的截面图可以看出, 经过蒸汽处理 后, $\mathrm{Si} / \mathrm{CNTs} / \mathrm{GP}$ 的石墨烯层间距明显增大, 层与层 一直不再是紧密堆叠的结构, 而是形成一种疏松、 褶皱、内部空隙丰富的片层结构。但是这样的疏松 二维结构并没有影响 $\mathrm{Si}$ 和 CNTs 在石墨烯层间的分 布, 两者依旧与石墨烯紧密连接, 保证了 $\mathrm{Si} / \mathrm{CNTs} /$ GP 负极材料整体导电网络的完整性, 且 $\mathrm{Si} / \mathrm{CNTs} /$ GP 仍具有良好的柔韧性和一定的力学强度, 可以 随意弯曲。

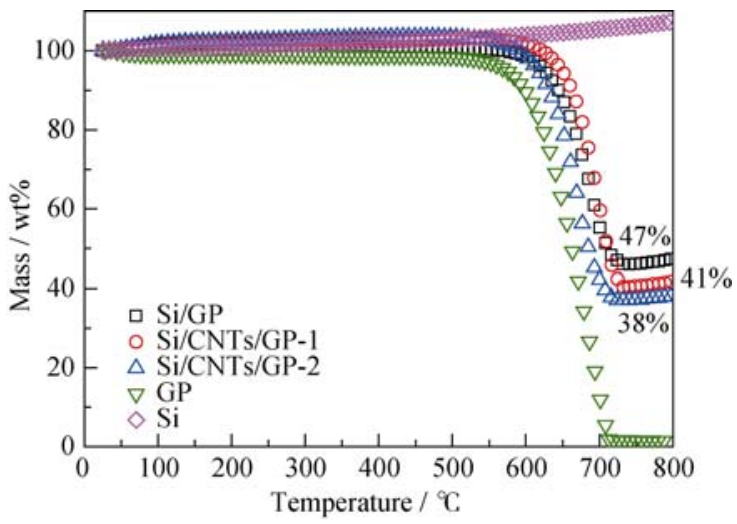

图 $6 \mathrm{Si} 、 \mathrm{GP} 、 \mathrm{Si} / \mathrm{GP}$ 和 $\mathrm{Si} / \mathrm{CNTs} / \mathrm{GP}-(1,2)$ 负极材料在空气 气氛中的热失重曲线

Fig. 6 TGA curves of Si, GP, Si/GP, and Si/CNTs/GP- $(1,2)$ composites in air

\section{3 石墨烯/硅自支撑材料的电化学性能}

图 8 为 $\mathrm{Si} / \mathrm{CNTs} / \mathrm{GP}-1$ 负极材料在 $2.0 \sim 0.01 \mathrm{~V}$ 电 压下的循环伏安曲线, 用来研究该负极材料在电池 充放电过程中发生的电化学反应。循环伏安曲线在 $0.01 \mathrm{~V}$ 处的还原峰对应锂离子与碳质基体的反应, $0.11 \mathrm{~V}$ 处的还原峰对应于 $\mathrm{Si}$ 与 $\mathrm{Li}$ 发生的嵌锂反应。 而 0.37 和 $0.54 \mathrm{~V}$ 处的氧化峰对应于 $\mathrm{C}-\mathrm{Li}$ 和 $\mathrm{Si}-\mathrm{Li}$ 的 脱锂反应。所有反应峰的强度都随着循环次数的增 加而变大，表明在循环充放电的驱动下，电解液进
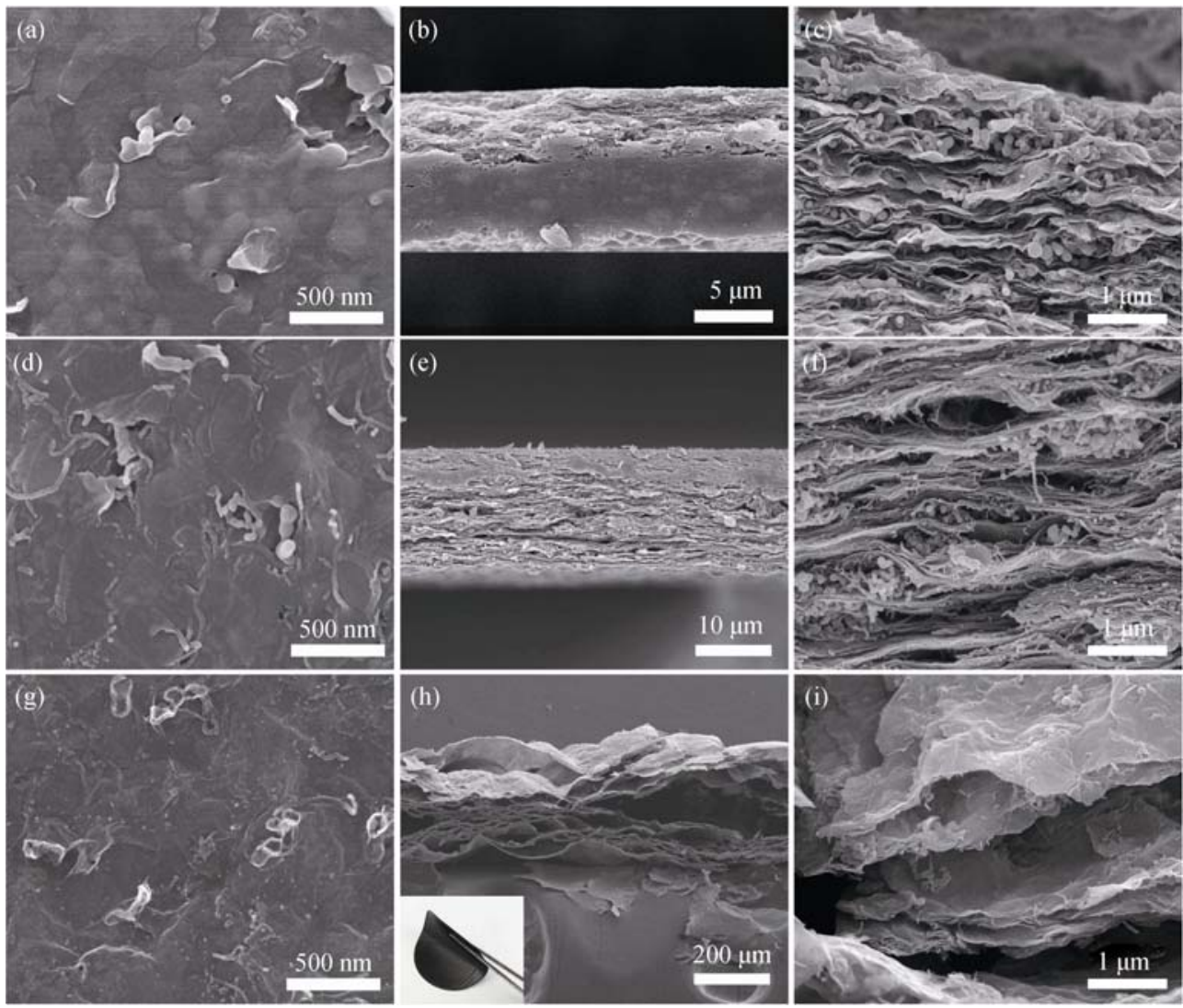

图 7 材料的表面和截面 FESEM 照片 $(a, b, c) \mathrm{Si} / \mathrm{GP} 、(\mathrm{~d}, \mathrm{e}, \mathrm{f}) \mathrm{Si} / \mathrm{CNTs} / \mathrm{GO} 、(\mathrm{~g}, \mathrm{~h}, \mathrm{i}) \mathrm{Si} / \mathrm{CNTs} / \mathrm{GP}$, 内图为实物图

Fig. 7 Surface and cross-section FESEM images of (a,b,c) Si/GP, (d, e, f) Si/CNTs/GO, and (g, h, i) Si/CNTs/GP with digital photo inset 


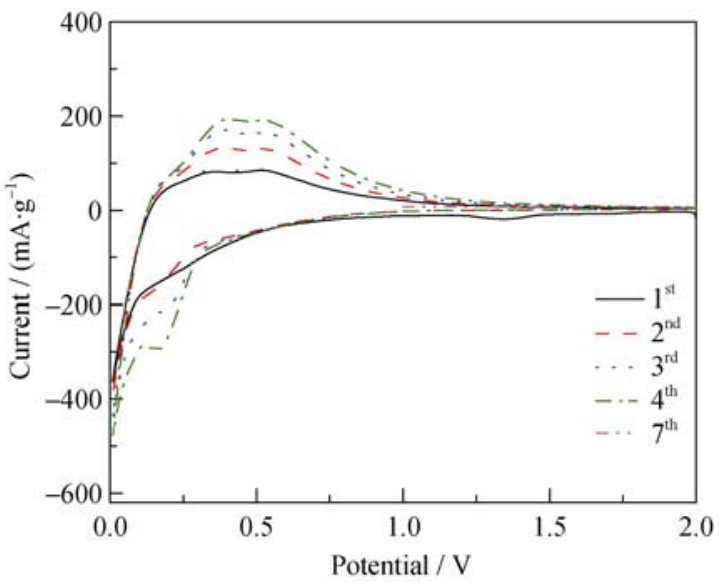

图 $8 \mathrm{Si} / \mathrm{CNTs} / \mathrm{GP}-1$ 负极材料的电池循环伏安曲线

Fig. $8 \mathrm{CV}$ curves of $\mathrm{Si} / \mathrm{CNTs} / \mathrm{GP}-1$ composite

一步浸润并活化负极材料。

图 9 对比了不同负极材料 $\mathrm{Si} / \mathrm{GP}$ 和 $\mathrm{Si} / \mathrm{CNTs} /$ GP- $(1,2)$ 在不同电流密度下的倍率循环稳定性。三 种负极材料依次在 $0.1 、 0.5 、 1 、 2 、 4 \mathrm{~A} / \mathrm{g}$ 电流密度 下充放电 10 个循环。低电流密度下, 负极材料的容 量与 $\mathrm{Si}$ 含量成正比, 且初始几次呈现缓慢增长的现 象, 这与 $\mathrm{CV}$ 曲线结果相匹配, 属于电池内部电解 液浸润和活化作用。当电流密度逐渐增大时, $\mathrm{Si} / \mathrm{GP}$ 的可逆容量衰减明显, 而 $\mathrm{Si} / \mathrm{CNTs} / \mathrm{GP}-(1,2)$ 的稳定 性相对较好。在 $4 \mathrm{~A} / \mathrm{g}$ 电流密度下, 三种负极材料的 可逆容量与 $\mathrm{Si}$ 含量呈反比, $\mathrm{Si} / \mathrm{CNTs} / \mathrm{GP}-2$ 的容量最 高为 $600 \mathrm{mAh} / \mathrm{g}$ 。这主要是由两个因素引起的：一 是 CNTs 可以在石墨烯片层之间相互贯穿形成网络 结构, 能够有效提高石墨烯片层的抗压能力。当 $\mathrm{Si}$ 与锂离子反应产生体积变化时, 石墨烯能够承受更 多的外界应力，保持材料整体结构稳定; 二是 $\mathrm{Si} / \mathrm{CNTs} / \mathrm{GP}-(1,2)$ 材料经过蒸汽处理后, 内部结构 变得非常疏松, 丰富的内部空隙可以为 $\mathrm{Si}$ 的体积变 化提供足够的空间，同时也有利于电解液的浸润， 缩短离子传输距离, 因此在高电流密度下表现出更 高的储锂容量和稳定性。Si/CNTs/GP-2 中 CNTs 的含 量要高于 $\mathrm{Si} / \mathrm{CNTs} / \mathrm{GP}-1$, 因此在 $4 \mathrm{~A} / \mathrm{g}$ 电流密度下, CNTs 表现出更明显的稳定结构的优势。当充放电电流 再次回到 $0.1 \mathrm{~A} / \mathrm{g}$ 时, Si/CNTs/GP- $(1,2)$ 两种负极材料能 够在 50 100 次循环中保持较好的稳定性, 最终可逆储 锂容量分别为 $1052 \mathrm{~mA} / \mathrm{g}$ 和 $1010 \mathrm{~mA} / \mathrm{g}$ 。而 $\mathrm{Si} / \mathrm{GP}$ 则衰 减严重, 说明 $\mathrm{Si} / \mathrm{GP}$ 经历过大电流充电后, 微观结构受 到了破坏，从而导致储锂容量的快速衰减。

图 10 为倍率循环测试之后 $\mathrm{Si} / \mathrm{GP}$ 和 $\mathrm{Si} / \mathrm{CNTs} /$ GP-1 负极极片的 FESEM 照片, 循环后 Si/GP 的表 面出现很多坑洼缺陷, 放大可以观察到明显的裂痕,

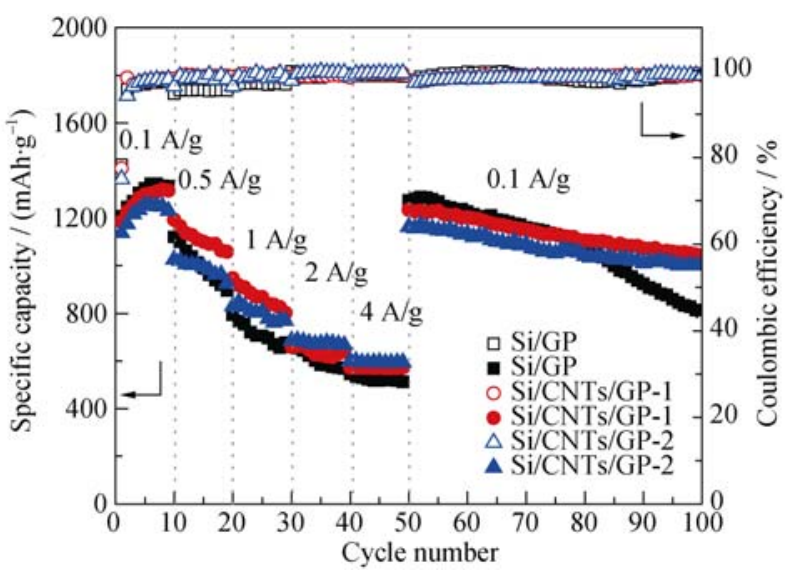

图 $9 \mathrm{Si} / \mathrm{GP}$ 和 $\mathrm{Si} / \mathrm{CNTs} / \mathrm{GP}-(1,2)$ 负极材料的电池倍率循环 性能

Fig. 9 Rate capacity of Si/GP and Si/CNTs/GP- $(1,2)$ at various rates as marked

如图 10(b)中白线及箭头所示。而 $\mathrm{Si} / \mathrm{CNTs} / \mathrm{GP}$ 负极 材料的结构与循环前相比基本一致，没有出现明显 的破坏及缺陷。经过 EDS 分析可以看出材料的元素 组成也未发生明显变化, 表明该负极材料在大电流 循环充放电过程中具有很好的结构稳定性。

为了进一步考察 $\mathrm{Si} / \mathrm{GP}$ 和 $\mathrm{Si} / \mathrm{CNTs} / \mathrm{GP}-(1,2)$ 负 极材料的电化学特性, 在倍率循环之后对电池进行 交流阻抗测试，如图 11 所示，从图中可以看出 Nyquist 曲线分为三个部分：高频区和中频区的半 圆，以及后面跟随的低频区的斜线。其中，高频区半 圆部分主要与电池内部接触电阻、SEI 膜电阻有关; 中频区半圆主要与电池内部电荷转移阻抗有关; 而 低频区斜线主要与锂离子在电极固相内的扩散过程 有关。通过 Zview 软件对三条 Nyquist 曲线进行模 拟得到一个合适的等效电路，如图 11 插图。其中 $R_{\mathrm{S}}$ 代表电池内部接触电阻, $R_{\mathrm{SEI}}$ 代表界面膜阻抗, $R_{\mathrm{ct}}$ 代表电荷转移阻抗, $W_{\mathrm{o}}$ 代表锂离子在固相内扩散的 Warburg 阻抗。模拟得到的具体数值见表 1 。从表中 可以看出, 三种负极材料的 $R_{\mathrm{s}}$ 变化不大, 而 $R_{\mathrm{SEI}}$ 和 $R_{\mathrm{ct}}$ 的变化却很明显。 $\mathrm{Si} / \mathrm{CNTs} / \mathrm{GP}-(1,2)$ 负极材料中 存在的 CNTs 能够有效完善材料的导电网络，提高 材料导电性，并且疏松的内部结构为电解液浸润、 锂离子的快速传输提供通道, 因此其传输阻力明显 减小, 这也与三种负极材料的倍率循环性能相一致。

\section{3 结论}

通过真空诱导自组装及静电吸附作用在石墨烯 中均匀引入 $\mathrm{Si}$ 纳米颗粒和 $\mathrm{CNTs}$, 再通过蒸汽处理 有效扩大石墨烯层层间距，得到结构疏松、导电网 

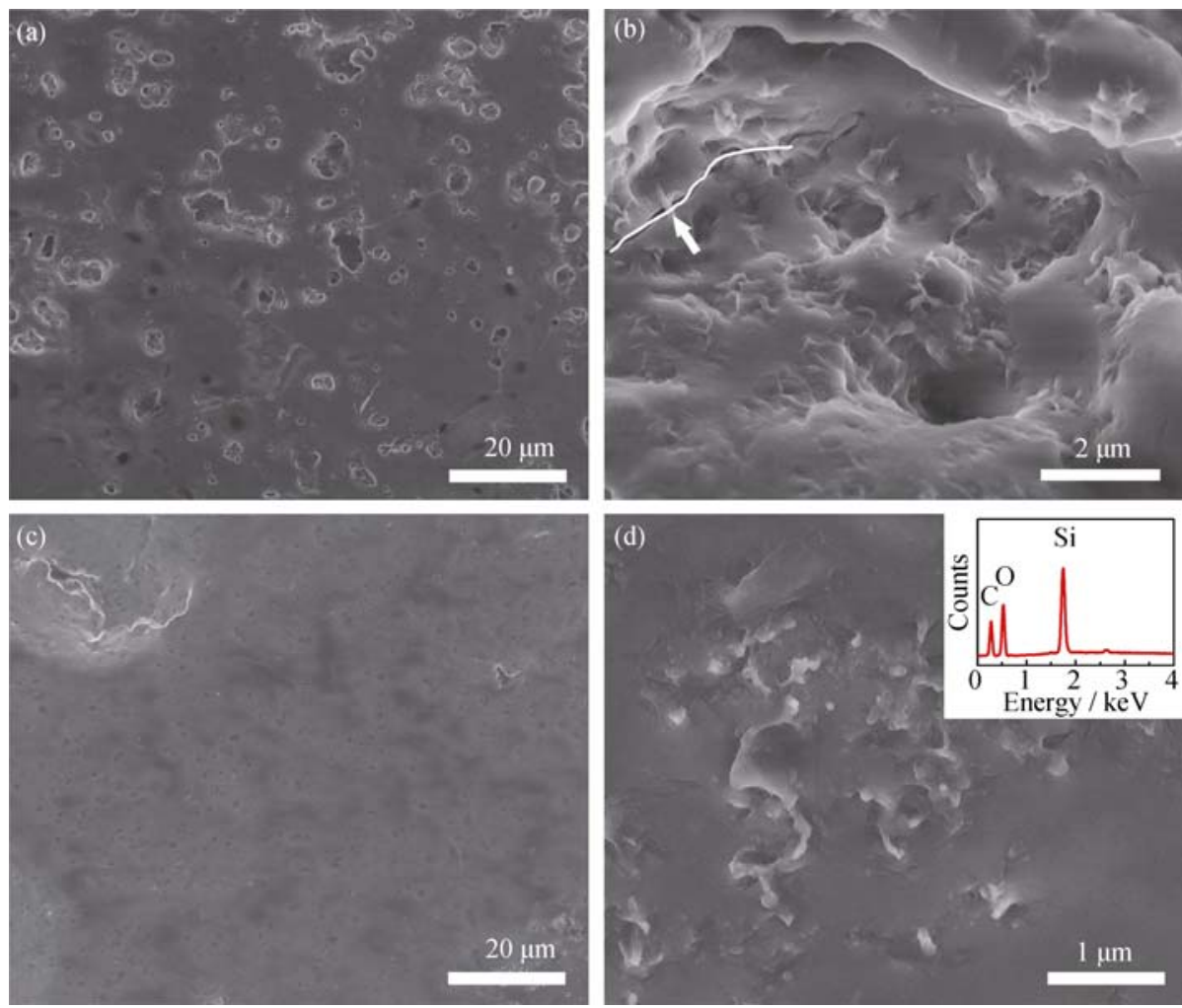

图 $10 \mathrm{Si} / \mathrm{GP}(\mathrm{a}, \mathrm{b})$ 和 $\mathrm{Si} / \mathrm{CNTs} / \mathrm{GP}-1(\mathrm{c}, \mathrm{d})$ 负极材料倍率循环后负极极片的 FESEM 照片及 EDS 能谱

Fig. 10 FESEM images of (a, b) Si/GP and (c, d) Si/CNTs/GP-1 anode after rate cycling test, with EDS inset

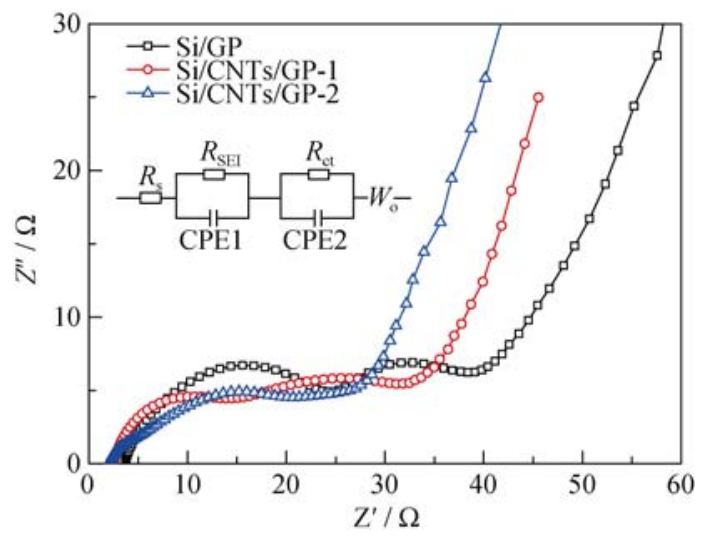

图 $11 \mathrm{Si} / \mathrm{GP}$ 和 $\mathrm{Si} / \mathrm{CNTs} / \mathrm{GP}-(1,2)$ 负极材料倍率循环后的交 流阻抗图

Fig. 11 Electrochemical impedance spectra of $\mathrm{Si} / \mathrm{GP}$ and $\mathrm{Si} / \mathrm{CNTs} / \mathrm{GP}$ composites after rate cycling test

The inset is the equivalent circuit

表 $1 \mathrm{Si} / \mathrm{GP}$ 和 Si/CNTs/GP-(1, 2)负极材料交流阻抗 拟合数据

Table 1 EIS fitting results of Si/GP and Si/CNTs/GP-(1, 2) electrodes

\begin{tabular}{cccc}
\hline Sample & $R_{\mathrm{s}} / \Omega$ & $R_{\mathrm{SEI}} / \Omega$ & $R_{\mathrm{ct}} / \Omega$ \\
\hline $\mathrm{Si} / \mathrm{GP}$ & 3.5 & 22.1 & 25.2 \\
$\mathrm{Si} / \mathrm{CNTs} / \mathrm{GP}-1$ & 2.5 & 8.7 & 19.9 \\
$\mathrm{Si} / \mathrm{CNTs} / \mathrm{GP}-2$ & 2.0 & 1.7 & 18.1 \\
\hline
\end{tabular}

络完整的 $\mathrm{Si} / \mathrm{CNTs} / \mathrm{GP}$ 自支撑膜，并以该材料作为 锂离子电池负极。该负极在 $4 \mathrm{~A} / \mathrm{g}$ 的大电流密度下 容量高达 $600 \mathrm{mAh} / \mathrm{g}$, 电流减小到 $0.1 \mathrm{~A} / \mathrm{g}$ 循环 50 次后容量仍有 $1010 \mathrm{mAh} / \mathrm{g}$, 表现出优异的大电流循 环稳定性能。这是因为蒸汽处理后 $\mathrm{Si} / \mathrm{CNTs} / \mathrm{GP}$ 存在 丰富的内部空穴和贯穿孔洞，有利于提高电解液对 负极的浸润性，极大缩短了锂离子的传输距离。同时 这些内部空穴也有利于缓冲 $\mathrm{Si}$ 充放电时的体积膨胀, 有效提高材料的结构稳定性和电化学性能。

\section{参考文献:}

[1] GOODENOUGH J B, KIM Y. Challenges for rechargeable Li batteries. Chem. Mater., 2010, 22(3): 587-603.

[2] DUNN B, KAMATH H, TARASCON J M. Electrical energy storage for the grid: a battery of choices. Science, 2011, 334(6058): 928-935.

[3] LI H, WANG Z, CHEN L, et al. Research on advanced materials for Li-ion batteries. Adv. Mater., 2009, 21(45): 4593-4607.

[4] TARASCON J M, ARMAND M. Issues and challenges facing rechargeable lithium batteries. Nature, 2001, 414(6861): 359-367.

[5] WANG H B, ZHOU Y H, TAO Z L, et al. Research progress of silicon-based anodes for lithium-ion batteries. Chinese J. Power Sources, 2009, 33(11): 1029-1032.

[6] GUO B K, SHU J, WANG Z X, et al. Electrochemical reduction of 
nano- $\mathrm{SiO}_{2}$ in hard carbon as anode material for lithium ion batteries. Electrochem. Commun., 2008, 10(12): 1876-1878.

[7] SUN Q, ZHANG B, FU Z W. Lithium electrochemistry of $\mathrm{SiO}_{2}$ thin film electrode for lithium-ion batteries. Appl. Surf. Sci., 2008, 254(13): 3774-3779.

[8] CHANG W S, PARK C M, KIM J H, et al. Quartz $\left(\mathrm{SiO}_{2}\right)$ : a new energy storage anode material for Li-ion batteries. Energy Environ. Sci., 2012, 5(5): 6895-6899.

[9] YU B C, HWA Y, KIM J H, et al. A new approach to synthesis of porous $\mathrm{SiO}_{x}$ anode for Li-ion batteries via chemical etching of $\mathrm{Si}$ crystallites. Electrochem. Acta, 2014, 117(4): 426-430.

[10] MA C L, MA C, WANG J Z, et al. Exfoliated graphite as a flexible and conductive support for Si-based $\mathrm{Li}$-ion battery anodes. Carbon, 2014, 72(3): 38-46.

[11] ZHOU X, YIN Y X, WAN L J, et al. Self-assembled nanocomposite of silicon nanoparticles encapsulated in graphene through electrostatic attraction for lithium-ion batteries. $A d v$. Energy Mater., 2012, 2(9): 1086-1090.

[12] SI Q, HANAI K, ICHIKAWA T, et al. A high performance silicon/carbon composite anode with carbon nanofiber for lithium-ion batteries. J. Power Sources, 2010, 195(6): 1720-1725.

[13] WANG L, DING C X, ZHANG L C, et al. A novel carbon-silicon composite nanofiber prepared via electrospinning as anode material for high energy-density lithium ion batteries. J. Power Sources, 2010, 195(15): 5052-5056.

[14] WU H, ZHENG G, LIU N, et al. Engineering empty space between Si nanoparticles for lithium-ion battery anodes. Nano Lett., 2012, 12(2): 904-909.

[15] JOHNSON D C, MOSBY J M, RIHA S C, et al. Synthesis of copper silicide nanocrystallites embedded in silicon nanowires for enhanced transport properties. J. Mater. Chem., 2010, 20(10): 1993-1998.

[16] LAÏK B, UNG D, CAILLARD A, et al. An electrochemical and structural investigation of silicon nanowires as negative electrode for Li-ion batteries. J. Solid State Electrochem., 2010, 14(10): $1835-1839$.

[17] CHEN H, XIAO Y, WANG L, et al. Silicon nanowires coated with copper layer as anode materials for lithium-ion batteries. J. Power Sources, 2011, 196(16): 6657-6662.

[18] HU L B, WU H, HONG S S, et al. Si nanoparticle-decorated Si nanowire networks for Li-ion battery anodes. Chem. Commun., 2011, 47(1): 367-369.

[19] PARK M H, KIM M G, JOO J, et al. Silicon nanotube battery anodes. Nano Lett., 2009, 9(11): 3844-3847.

[20] LEE W J, PARK M H, WANG Y, et al. Nanoscale Si coating on the pore walls of $\mathrm{SnO}_{2}$ nanotube anode for Li rechargeable batteries. Chem. Commun., 2010, 46(4): 622-624.
[21] SONG T, XIA J L, LEE J H, et al. Arrays of sealed silicon nanotubes As anodes for lithium ion batteries. Nano Lett., 2010, 10(5): $1710-1716$.

[22] YU Y, GU L, ZHU C, et al. Reversible storage of lithium in silver-coated three-dimensional macroporous silicon. Adv. Mater, 2010, 22(20): 2247-2250.

[23] YAO Y, MCDOWELL M T, RYU I, et al. Interconnected silicon hollow nanospheres for lithium-ion battery anodes with long cycle life. Nano Lett., 2011, 11(7): 2949-2954.

[24] CHEN D, MEI X, JI G, et al. Reversible lithium-ion storage in silver-treated nanoscale hollow porous silicon particles. Angew. Chem. Int. Ed. Engl., 2012, 51(10): 2409-2413.

[25] BAI X, WANG B, WANG H, et al. In situ synthesis of carbon fiber-supported $\mathrm{SiO}_{x}$ as anode materials for lithium ion batteries. RSC Adv., 2016, 6(39): 32798-32803.

[26] LEE B S, SON S B, PARK K M, et al. Fabrication of Si core/C shell nanofibers and their electrochemical performances as a lithium-ion battery anode. J. Power Sources, 2012, 206(2): 267-273.

[27] LIU X H, ZHANG J, SI W P, et al. Sandwich nano architecture of $\mathrm{Si} /$ reduced graphene oxide bilayer nanomembranes for $\mathrm{Li}$-ion batteries with long cycle life. ACS Nano, 2015, 9(2): 1198-1205.

[28] ZHAO X, HAYNER C M, KUNG M C, et al. In-plane vacancy-enabled high-power Si-graphene composite electrode for lithium-ion batteries. Adv. Energy Mater., 2011, 1(6): 1079-1084.

[29] MAGASINSKI A, DIXON P, HERZBERG B, et al. Highperformance lithium-ion anodes using a hierarchical bottom-up approach. Nature Mater., 2010, 9(4): 353-358.

[30] BAI X J, YU Y Y, KUNG H H, et al. Si@ $\mathrm{SiO}_{x} /$ graphene hydrogel composite anode for lithium-ion battery. J. Power Sources, 2016, 306: $42-48$.

[31] ZHANG S, SHAO Y Y, LIAO H G, et al. Polyelectrolyte-induced reduction of exfoliated graphite xxide: a facile route to synthesis of soluble graphene nanosheets. ACS Nano, 2011, 5(3): 1785-1791.

[32] ZHANG J, JIANG J, ZHAO X S. Synthesis and capacitive properties of manganese oxide nanosheets dispersed on functionalized graphene Sheets. J. Phys. Chem. C, 2011, 115(14): 6448-6454.

[33] ZHU Y C, ZHOU Y H, YU L Y, et al. A highly stable and active Pd catalyst on monolithic cordierite with graphene coating assisted by PDDA. RSC Adv., 2014, 4(19): 9480-9483.

[34] ZHANG H J, GAI P B, CHENG R, et al. Self-assembly synthesis of a hierarchical structure using hollow nitrogen-doped carbon spheres as spacers to separate the reduced graphene oxide for simultaneous electrochemical determination of ascorbic acid, dopamine and uric acid. Anal. Methods, 2013, 5(14): 3591-3600.

[35] WANG D W, MIN Y G, YU Y H, et al. A general approach for fabrication of nitrogen-doped graphene sheets and its application in supercapacitors. J. Colloid and Interf. Sci., 2014, 417(3): 270-277. 